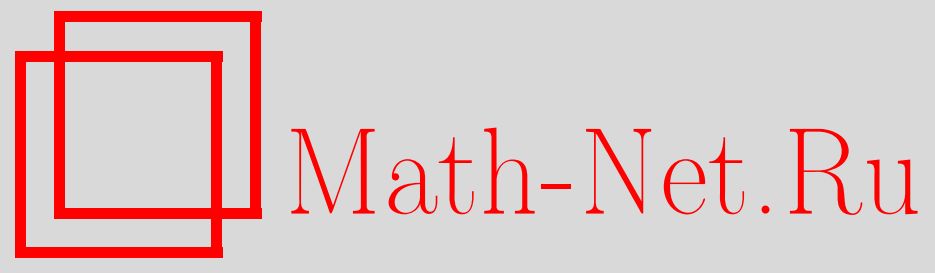

В. Д. Бейбалаев, А. А. Аливердиев, Р. А. Магомедов, Р. Р. Мейланов, Э. Н. Ахмедов, Моделирование процессов промерзания одномерным уравнением теплопроводности с операторами дробного дифференцирования, Вестн. Сам. гос. техн. ун-та. Сер. Физ.-мат. науки, 2017, номер 2, 376-387

DOI: https://doi.org/10.14498/vsgtu1492

Использование Общероссийского математического портала MathNet.Ru подразумевает, что вы прочитали и согласны с пользовательским соглашением

http://www . mathnet.ru/rus/agreement

Параметры загрузки:

IP: 54.172 .240 .79

26 апреля 2023 г., 15:41:35

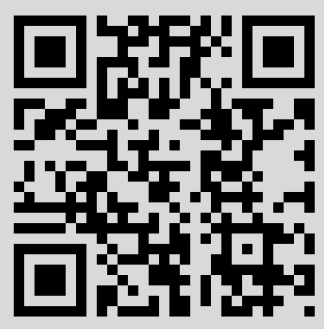


УДК 517.958:536.2

\title{
Моделирование процессов промерзания одномерным уравнением теплопроводности с операторами дробного дифференцирования
}

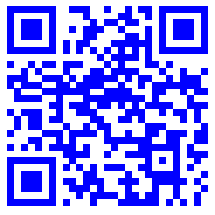

\author{
В. Д. Бейбалаев ${ }^{1,2}$, А. А. Аливердиев ${ }^{1,2}$, \\ P. А. Магомедов ${ }^{2}$, Р. Р. Мейланов ${ }^{2}$, Э. Н. Ахмедов ${ }^{2}$ \\ 1 Дагестанский государственный университет, \\ Россия, 367025, Махачкала, ул. М. Гаджиева, 43а. \\ 2 Институт проблем геотермии Дагестанского НЦ РАН, \\ Россия, 367030, Махачкала, пр. Шамиля, 39а.
}

\section{Аннотация}

В работе исследована задача Стефана в обобщении для фрактальных сред с применением аппарата производных дробного порядка в смысле Капуто по времени. Построена разностная схема. Разработан алгоритм и создана программа численного решения задачи Стефана с оператором дробного дифференцирования. Для начальных условий и параметров замерзающего грунта получены зависимости температурного поля от координаты и времени при различных значениях дробного параметра $\alpha$. Оценены функциональные зависимости движения межфазной границы для обобщенного условия Стефана в зависимости от значения $\alpha$. Установлено, что с уменьшением $\alpha$ процесс промерзания замедляется.

Ключевые слова: дробная производная по Капуто, фрактальная структура, задача Стефана, эффект памяти, разностная схема, теплопроводность, фазовый переход, фазовая граница.

Получение: 28 апреля 2016 г. / Исправление: 10 апреля 2017 г. / Принятие: 12 июня 2017 г. / Публикация онлайн: 4 июля 2017 г.

\section{Статья}

2 () Контент публикуется на условиях лицензии Creative Commons Attribution 4.0 International (https://creativecommons.org/licenses/by/4.0/deed.ru)

\section{Образец для цитирования}

Бейбалаев В.Д., Аливердиев А. А., Магомедов Р. А., Мейланов Р. Р., А хмедо в Э. Н. Моделирование процессов промерзания одномерным уравнением теплопроводности с операторами дробного дифференцирования // Вестн. Сам. гос. техн. унта. Сер. Физ.-мат. науки, 2017. Т. 21, № 2. С. 376-387. doi: 10.14498/vsgtu1492.

\section{Сведения об авторах}

Ветлугин Джабраилович Бейбалаев (10) http://orcid.org/0000-0002-4881-9264

кандидат физико-математических наук, доцент; доцент, каф. прикладной математики $^{1}$; старший научный сотрудник, лаб. математического моделирования геотермальных объектов ${ }^{2}$; e-mail: kaspij_03@mail.ru

\section{Абутраб Александрович Аливердиев}

доктор физико-математических наук, профессор; профессор, каф. теоретической и математической физики ${ }^{1}$; заведующий лабораторией, лаб. математического моделирования геотермальных объектов ${ }^{2}$; e-mail: aliverdi@mail.ru

Рамазан Абдуллаевич Магомедов (D) http://orcid.org/0000-0001-6224-6215

старший научный сотрудник; лаб. математического моделирования геотермальных объектов; e-mail: ramazan_magomedov@rambler.ru 
Введение. Задача Стефана описывает явления тепломассопереноса в средах с фазовым переходом, сопровождающимся выделением или поглощением тепла. Большой прикладной интерес представляет обобщение задачи Стефана для сред, в которых не выполняется принцип локального равновесия, что приводит к необходимости учета особенностей теплопереноса на межфазной границе с учетом нелокальных эффектов по времени (эффект памяти) [1-3] и по пространству (эффект пространственных корреляций) [3, 4].

Одно из направлений обобщения неравновесной термодинамики связано с развитием концепции фрактала. Процессы переноса тепла в этом случае могут быть описаны на основе дифференциальных уравнений в производных дробного порядка $[5,6]$.

Ранее [3] нами было показано, что учет эффектов памяти приводит к зависимости координаты межфазной границы от времени. В настоящей работе нами была поставлена задача сравнить пространственно-временные температурные поля, получающиеся при варьировании дробного параметра $\alpha$ для производной по времени (производная Капуто). В дальнейшем сопоставление с экспериментальными результатами позволит определить данный коэффициент для различных грунтов.

1. Математическая постановка задачи. Влажная фрактальная структура находится с некоторой постоянной температурой $T_{0}$ в талом состоянии. Внезапно в начальный момент времени на поверхности устанавливается температура $T_{\mathrm{c}}$, которая ниже температуры замерзания $T_{3}$. При этом с некоторой переменной толщиной $\xi=f(t)$ образуется промерзший слой. Нижняя подвижная граница имеет всегда температуру замерзания $T_{3}$, а на границе происходит фазовый переход, на что требуется теплота фазового перехода $Q_{f}$. При этом верхняя граница талой зоны имеет постоянную температуру замерзания, а нижняя - температуру грунта на большой глубине.

В качестве математической модели процесса промерзания во фрактальных структурах рассмотрим следующую задачу:

$$
\begin{gathered}
\partial_{0 t}^{\alpha} T_{1}(x, t)=D_{1} \frac{\partial^{2} T_{1}(x, t)}{\partial x^{2}}, \quad 0<x<\xi(t), \quad t>0 \\
\partial_{0 t}^{\alpha} T_{2}(x, t)=D_{2} \frac{\partial^{2} T_{2}(x, t)}{\partial x^{2}}, \quad \xi(t)<x<L, \quad t>0 \\
T(x, 0)=T_{0}, \\
T(0, t)=T_{\mathrm{c}}, \quad \frac{\partial T(L, t)}{\partial x}=0, \quad t>0, \\
x=\xi(t):\left\{\begin{array}{l}
T_{1}=T_{2}=T_{3}, \\
\lambda_{1} \frac{\partial T_{1}}{\partial x}-\lambda_{2} \frac{\partial T_{2}}{\partial x}=Q_{f} \partial_{0 t}^{\alpha} \xi(t)
\end{array}\right.
\end{gathered}
$$

Рашид Русланович Мейланов (D) http://orcid.org/0000-0001-9791-2142 младший научный сотрудник; лаб. математического моделирования геотермальных объектов

Энвер Нариманович Ахмедов (D) http://orcid.org/0000-0003-4829-6034 младший научный сотрудник; лаб. математического моделирования геотермальных объектов; e-mail: aen-code@yandex .ru 
где $0<\alpha \leqslant 1, \rho$ - плотность грунта, $t=\tau / \tau_{0}, x=\xi / \xi_{0}-$ безразмерные время и координата, $\tau_{0}, \xi_{0}$-характерные время и масштаб, $D_{1}=a_{1} \tau_{0} / \xi_{0}^{2}$, $D_{2}=a_{2} \tau_{0} / \xi_{0}^{2}$ - безразмерные коэффициенты температуропроводности, $Q-$ количество тепла, выделяемое или поглощаемое в процессе таяния льда или замерзания воды, постоянные температуры $T_{\mathrm{c}}<T_{3}<T_{0}$,

$$
\partial_{0 t}^{\alpha} T(x, t)=\frac{1}{\Gamma(1-\alpha)} \int_{0}^{t} \frac{T_{t}^{\prime}(x, s)}{(t-s)^{\alpha}} d s
$$

- частная дробная производная Капуто.

Как известно, при поиске аналитических решений дробных дифференциальных уравнений возникают большие трудности. Поэтому в настоящее время при решении дробных дифференциальных уравнений применяют как аналитические, так и численные методы.

Численным методам решения краевых задач для дифференциальных уравнений с производными дробного порядка посвящены работы [6-13].

Унифицированным методом приближенного решения дифференциальных уравнений, применимым для широкого класса уравнений математической физики, является метод конечных разностей (или метод сеток) [14].

2. Численная математическая модель. Для решения задачи (1), (2) методом сеток вводим равномерную сетку по пространственной переменной

$$
x_{m}=m h, \quad m=0,1, \ldots, M, \quad h=L / N,
$$

и неравномерную сетку по времени

$$
t_{n+1}=t_{n}+\tau_{n+1}, \quad n=0,1, \ldots, N-1, \quad t_{0}=0, \quad t_{N}=t_{\mathrm{KOH}}, \quad \tau_{n+1}>0 .
$$

Для дробной производной Капуто имеет место аппроксимация [15]

$$
\partial_{0 t}^{\alpha} T\left(x_{m}, t_{n}\right)=\frac{1}{\Gamma(2-\alpha) \tau_{n}} \sum_{k=0}^{n}\left(T_{1, m}^{k+1}-T_{1, m}^{k}\right)\left(t_{n-k+1}^{1-\alpha}-t_{n-k}^{1-\alpha}\right)+O(\tau),
$$

а для производной $\partial^{2} T(x, t) / \partial x^{2}$ имеет место аппроксимация

$$
\frac{\partial^{2} T\left(x_{m}, t_{n}\right)}{\partial x^{2}}=\frac{T_{m+1}^{n+1}-2 T_{m}^{n+1}+T_{m-1}^{n+1}}{h^{2}}+O\left(h^{2}\right) .
$$

Шаг по времени $\tau_{n+1}, n=0,1, \ldots, N-1$, нужно выбрать таким образом, чтобы за этот временной промежуток (от $t_{n}$ до $t_{n+1}$ ) граница фазового перехода сдвинулась ровно на один шаг пространственной сетки. Тогда, воспользовавшись разностной аппроксимацией дробной производной (3), можно записать

$$
\begin{aligned}
\partial_{0 t}^{\alpha} \xi(t) \approx \frac{1}{\Gamma(2-\alpha) \tau_{n+1}} & \sum_{k=0}^{n}\left(\xi_{k+1}-\xi_{k}\right)\left(t_{n-k+1}^{1-\alpha}-t_{n-k}^{1-\alpha}\right) \approx \\
& \approx \frac{h}{\Gamma(2-\alpha) \tau_{n+1}} \sum_{k=0}^{n}\left(t_{n-k+1}^{1-\alpha}-t_{n-k}^{1-\alpha}\right)=\frac{h t_{n}^{1-\alpha}}{\Gamma(2-\alpha) \tau_{n+1}} .
\end{aligned}
$$


Воспользовавшись (3), (4), получим следующую разностную схему:

$$
\begin{array}{r}
\frac{1}{\Gamma(2-\alpha) \tau_{n}} \sum_{k=0}^{n}\left(T_{1, m}^{k+1}-T_{1, m}^{k}\right)\left(t_{n-k+1}^{1-\alpha}-t_{n-k}^{1-\alpha}\right)=\frac{D_{1}}{h^{2}}\left(T_{1, m+1}^{n+1}-2 T_{1, m}^{n+1}+T_{1, m-1}^{n+1}\right) \\
m=1,2, \ldots, m^{*}-1,\left.\quad T_{1}\right|_{m=1}=T_{\mathrm{c}},\left.\quad T_{1}\right|_{m=m^{*}}=T_{3}, \quad \text { (5) }
\end{array}
$$

где $m=m^{*}$ - граница фазового перехода.

Тогда разностная схема второй части уравнения будет иметь вид

$$
\begin{array}{r}
\frac{1}{\Gamma(2-\alpha) \tau_{n}} \sum_{k=0}^{n}\left(T_{2, m}^{k+1}-T_{2, m}^{k}\right)\left(t_{n-k+1}^{1-\alpha}-t_{n-k}^{1-\alpha}\right)=\frac{D_{2}}{h^{2}}\left(T_{2, m+1}^{n+1}-2 T_{2, m}^{n+1}+T_{2, m-1}^{n+1}\right) \\
m=m^{*}+1, \ldots, M-1,\left.\quad T_{2}\right|_{m=m^{*}}=T_{3},\left.\quad \frac{\partial T_{2}}{\partial x}\right|_{m=M}=0 . \quad \text { (6) }
\end{array}
$$

После преобразований уравнения (5) и (6) соответственно запишутся следующим образом:

$$
\begin{aligned}
& A_{m} T_{1, m-1}^{n+1}-C_{m} T_{1, m}^{n+1}+B_{m} T_{1, m+1}^{n+1}=F_{m} \\
& m=1,2, \ldots, m^{*}-1,\left.\quad T_{1}\right|_{m=1}=T_{c},\left.\quad T_{1}\right|_{m=m^{*}}=T_{3},
\end{aligned}
$$

где

$$
\begin{gathered}
A_{m}=B_{m}=\frac{D_{1}}{h^{2}}, \quad C_{m}=\frac{2 D_{1}}{h^{2}}+\frac{t_{1}^{1-\alpha}}{\Gamma(2-\alpha) \tau_{n}} \\
F_{m}=-\frac{t_{1}^{1-\alpha}}{\Gamma(2-\alpha) \tau_{n}} T_{1, m}^{n}+\frac{t_{1}^{1-\alpha}}{\Gamma(2-\alpha) \tau_{n}} \sum_{k=0}^{n-1}\left(T_{1, m}^{k+1}-T_{1, m}^{k}\right)\left(t_{n-k+1}^{1-\alpha}-t_{n-k}^{1-\alpha}\right)
\end{gathered}
$$

и

$$
\begin{aligned}
& A_{m} T_{1, m-1}^{n+1}-C_{m} T_{2, m}^{n+1}+B_{m} T_{2, m+1}^{n+1}=F_{m} \\
& m=m^{*}+1, \ldots, M-1,\left.\quad T_{2}\right|_{m=m^{*}}=T_{3},\left.\quad \frac{\partial T_{2}}{\partial x}\right|_{m=M}=0
\end{aligned}
$$

где

$$
\begin{gathered}
A_{m}=B_{m}=\frac{D_{2}}{h^{2}}, \quad C_{m}=\frac{2 D_{2}}{h^{2}}+\frac{t_{1}^{1-\alpha}}{\Gamma(2-\alpha) \tau_{n}} \\
F_{m}=-\frac{t_{1}^{1-\alpha}}{\Gamma(2-\alpha) \tau_{n}} T_{2, m}^{n}+\frac{t_{1}^{1-\alpha}}{\Gamma(2-\alpha) \tau_{n}} \sum_{k=0}^{n-1}\left(T_{2, m}^{k+1}-T_{2, m}^{k}\right)\left(t_{n-k+1}^{1-\alpha}-t_{n-k}^{1-\alpha}\right) .
\end{gathered}
$$

Проведем дискретизацию граничного условия в случае $x=\xi(t)$ :

$$
\lambda_{1} \frac{T_{1, m^{*}}-T_{1, m^{*}-1}}{h}-\lambda_{2} \frac{T_{2, m^{*}+1}-T_{2, m^{*}}}{h}=Q_{f} \frac{h t_{n}^{1-\alpha}}{\Gamma(2-\alpha) \tau_{n+1}}
$$

то есть

$$
\lambda_{1} \frac{T_{3}-T_{1, m^{*}-1}}{h}-\lambda_{2} \frac{T_{2, m^{*}+1}-T_{3}}{h}=Q_{f} \frac{h t_{n}^{1-\alpha}}{\Gamma(2-\alpha) \tau_{n+1}} .
$$


Таким образом,

$$
\tau_{n+1}=\frac{Q_{f} h^{2} t_{n}^{1-\alpha}}{\left(\lambda_{1}\left(T_{3}-T_{m^{*}-1}\right)-\lambda_{2}\left(T_{2, m^{*}+1}-T_{3}\right)\right) \Gamma(2-\alpha)} .
$$

Шаг по времени зависит от температуры. Поэтому поле температуры можно определить методом простой итерации.

Решение систем (7) и (10), где коэффициенты определяются согласно равенствам (8), (9) и (11), (12), будем на каждом временном слое находить методом прогонки в виде

$$
T_{m}=Q_{m+1} T_{m+1}+D_{m+1}, \quad m=M-1, M-2, \ldots, 0,
$$

где

$Q_{m+1}=\frac{B_{m}}{C_{m}-Q_{m} A_{m}}, \quad D_{m+1}=\frac{A_{m} C_{m}-F_{m}}{C_{m}-Q_{m} A_{m}}, \quad m=1,2, \ldots, M-1$.

АЛГОРИТМ ЧИСЛЕННОГО РЕШЕНИЯ ЗАДАЧИ

1) Вводим входные данные.

2) Находим расчетный шаг сетки по координате $h=L / M$.

3) Вводим начальное поле температуры $T_{m}^{0}, m=1,2, \ldots, M$.

4) Задаем начальное положение границы фазового перехода $k=1$.

5) Если время $t \geqslant t_{\text {кон }}$, то переходим к пункту 17 , иначе - к пункту 6.

6) Сохраняем температурное поле с предыдущего временного слоя:

$$
T_{m}^{n}=T_{m}, \quad m=1,2, \ldots, M
$$

7) Задаем положение границы фазового перехода $k=k+1$.

8) Сохраняем в новом векторе поле температуры с предыдущей итерации:

$$
T_{m}^{s}=T_{m}^{n}, \quad m=1,2, \ldots, M
$$

9) Определяем шаг по времени по формуле (13).

10) Задавая прогоночные коэффициенты $Q_{1}$ и $D_{1}$ на основе левого граничного условия, согласно (15) находим прогоночные коэффициенты.

11) Задаем температурное поле на границе фазового перехода $T_{k}^{s+1}=T_{3}$.

12) Находим температурное поле до фазовой границы $T_{m}^{s+1}, m=k-1, \ldots$, 2,1, по формуле (14).

13) Задавая прогоночные коэффициенты $Q_{1}$ и $D_{1}$ на основе левого граничного условия, согласно (15) находим прогоночные коэффициенты.

14) Находим температуру на правой границе $T_{M}^{s+1}$ на основе правого граничного условия.

15) Находим температурное поле после фазовой границы $T_{m}^{s+1}, m=M-1$, $\ldots, k$ по формуле (14).

16) Если $\max _{m}\left|T_{m}^{s+1}-T_{m}^{s}\right| \leqslant \varepsilon$, то увеличиваем переменную времени на шаг $\tau$ и переходим к пункту 6 , иначе переходим к пункту 9.

17) Выводим результаты. 
3. Результаты и их обсуждение. Для анализа полученного решения нами были выбраны начальные условия, типичные для зимнего промерзания, и взяты табличные теплофизические характеристики [15, стр. 133]: глубина грунта $L=0.3 \mathrm{M}$, теплофизические характеристики промерзшей зоны грунта $-\lambda_{1}=2.7 \mathrm{Bт} /(\mathrm{m} \cdot \mathrm{K}), \rho_{1}=917 \mathrm{kг} / \mathrm{m}^{3}, a_{1}=2090$ Дж/(кг $\left.\cdot \mathrm{K}\right)$; теплофизические характеристики талой зоны грунта: $\lambda_{2}=0.6 \mathrm{BT} /(\mathrm{M} \cdot \mathrm{K}), \rho_{2}=1000 \mathrm{kг} / \mathrm{m}^{3}$, $a_{2}=4220$ Дж/(кг $\left.\mathrm{K}\right)$; характерные температуры $T_{0}=293 \mathrm{~K}, T_{3}=273 \mathrm{~K}$, $T_{\mathrm{c}}=268 \mathrm{~K}$, теплота фазового перехода $Q_{\phi}=3.32 \cdot 10^{5}$ Дж/кг, влажность грунта $w=1 \mathrm{kг} / \mathrm{kг.}$

На рис. 1 приведены графики численного решения задачи при различных значениях параметра $\alpha$ и в произвольно выбранный момент времени $t=130000$ сек. Как видно из рисунка, в точке замерзания $\left(0^{\circ} \mathrm{C}\right)^{1}$ температурная зависимость претерпевает излом, причем с уменьшением показателя дробной производной точка замерзания лежит ближе к поверхности.

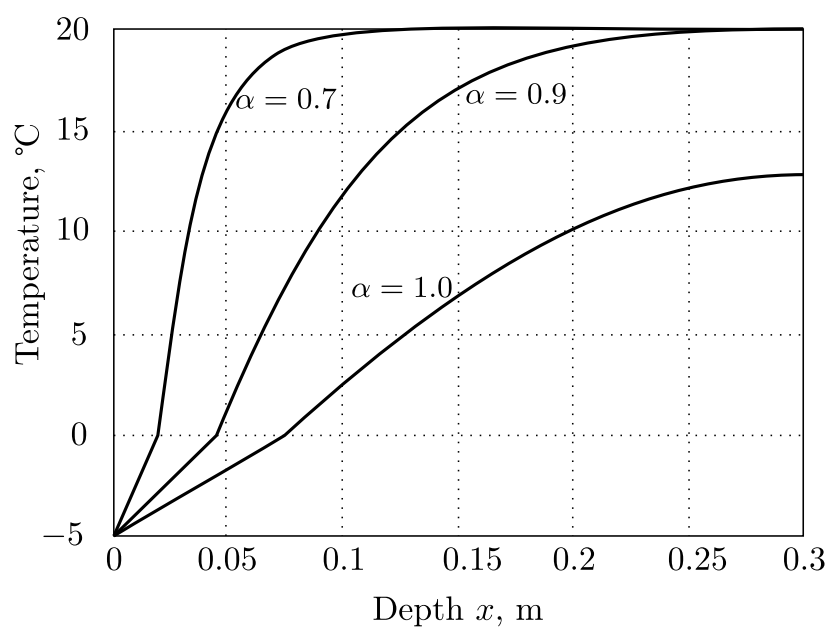

Рис. 1. Графики численного решения задачи (1), (2) при различных значениях параметра $\alpha$ в момент времени $t=130000$ сек [Figure 1. Graphs of the numerical solution of the problem

(1), (2) for different values of the parameter $\alpha$ at time $t=130000 \mathrm{sec}]$

На рис. 2 и 3 приведены графики зависимости температуры от времени на глубинах 0.0015 м и 0.075 м при различных значениях параметра дробной производной. Как видно из рисунков, промерзание на глубине 0.0015 м происходит практически сразу, в то же время на глубине 0.075 м при любом значении $\alpha$ температура достаточно долго держится выше точки замерзания.

В обоих случаях даже незначительное уменьшение $\alpha$ приводит к существенному замедлению скорости охлаждения, что характерно для сред с фрактальной структурой [2].

В таблице приведены моменты времени, соответствующие фазовым переходам, для различных координат при значениях параметра $\alpha=1$ и $\alpha=0.9$.

Данные для $\alpha=1$ и $\alpha=0.9$ соответствуют функциональной зависимости $\xi(t) \approx 0.00015 t^{1 / 2}$ и $\xi(t) \approx 0.0002 t^{0.45}$ соответственно. Таким образом, фазовую границу можно задать функциональной зависимостью $\xi(t) \approx \sigma(\alpha) t^{\alpha / 2}$, где $0<\alpha \leqslant 1$.

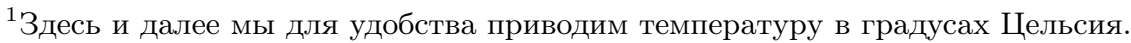




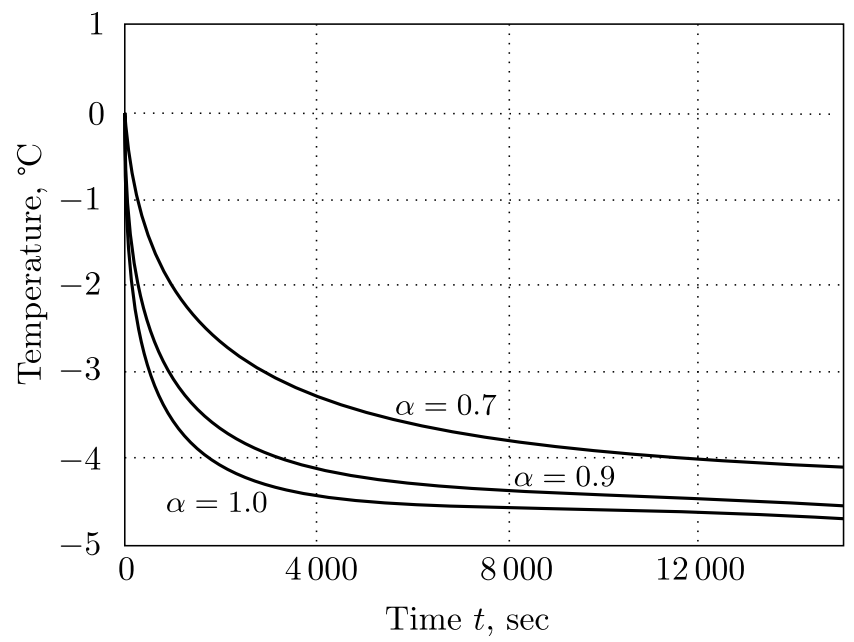

Рис. 2. Графики зависимости температуры от времени на глубине 0.0015 м в различные моменты времени и при различных значениях параметра $\alpha$ и $T_{\mathrm{c}}=-5^{\circ} \mathrm{C}$ [Figure 2. Graphs of temperature versus time at a depth of $0.0015 \mathrm{~m}$ at different times and for different values of the parameter $\alpha$ and $\left.T_{\mathrm{c}}=-5^{\circ} \mathrm{C}\right]$

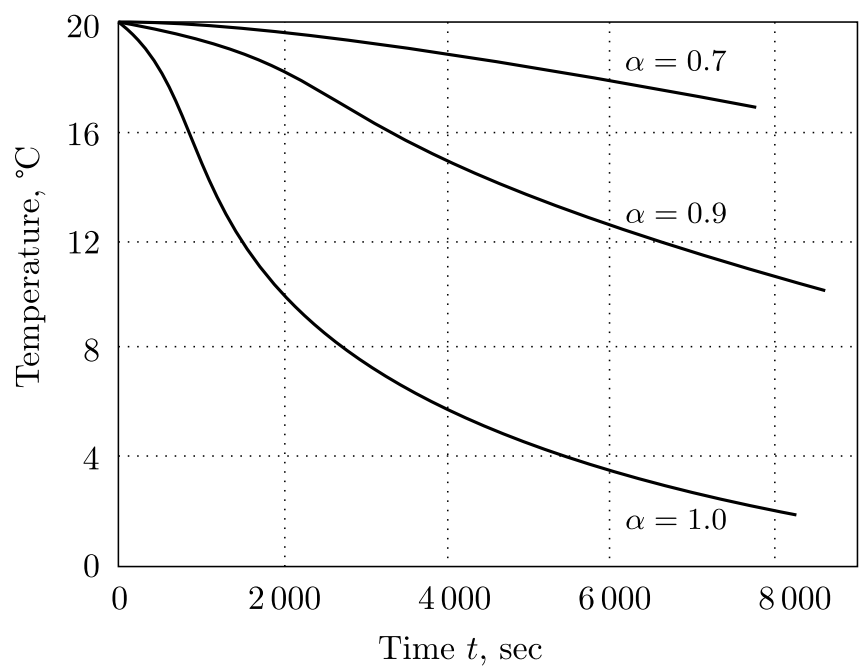

Рис. 3. Графики зависимости температуры от времени на глубине 0.075 м при различных значениях параметра $\alpha$ и $T_{\mathrm{c}}=-5^{\circ} \mathrm{C}$ [Fig. 3. Graphs of temperature versus time at a depth of $0.075 \mathrm{~m}$ for different values of the parameter $\alpha$ and $\left.T_{\mathrm{c}}=5^{\circ} \mathrm{C}\right]$

Time corresponding to phase transitions

\begin{tabular}{l||c|c|c|c}
\hline Depth $x, \mathrm{~m}$ & 0.0015 & 0.0030 & 0.0045 & 0.0060 \\
\hline Time $t, \sec (\alpha=1)$ & 93 & 296 & 606 & 1023 \\
\hline Time $t, \sec (\alpha=0.9)$ & 154 & 545 & 1190 & 2105 \\
\hline
\end{tabular}

Для скорости движения межфазной границы, согласно обобщенному условию Стефана, имеем следующее выражение:

$$
V_{\Gamma}=\partial_{0 t}^{\alpha} \xi(t)=\frac{\sigma}{\Gamma(1-\alpha)} \int_{0}^{t} \frac{\xi^{\prime}(s)}{(t-s)^{\alpha}} d s=\frac{\sigma(\alpha) \Gamma(\alpha / 2+1)}{\Gamma(1-\alpha / 2)} t^{-\alpha / 2} .
$$


Таким образом, скорость движения фазовой границы является функцией, зависящей от времени и параметра производной дробного порядка. Фазовая скорость $V_{\Gamma} \rightarrow 0$ при $t \rightarrow \infty$.

Заключение. В работе на основе классической модели Стефана построена математическая модель процессов промерзания с учетом особенностей теплопереноса на межфазовой границе, учитывающая эффекты памяти и фрактальность среды. Разработан алгоритм и создана программа численного решения задачи Стефана с оператором дробного дифференцирования. Оценены функциональные зависимости движения межфазной границы для обобщенного условия Стефана в зависимости от значения дробного параметра $\alpha$. Установлено, что переход к дробным производным позволяет описать замедление процесса промерзания грунта относительно классического решения.

Конкурирующие интересы. Мы не имеем конкурирующих интересов.

Авторский вклад и ответственность. Все авторы принимали участие в разработке концепции статьи и в написании рукописи. Авторы несут полную ответственность за предоставление окончательной рукописи в печать. Окончательная версия рукописи была одобрена всеми авторами.

Финансирование. Работа выполнена при частичной поддержке РФФИ (грант № 16 08-00067_a).

\section{Библиографический список}

1. Liu Junyi, Xu Mingyu Some exact solutions to Stefan problems with fractional differential equations // J. Math. Anal. Appl., 2009. vol.351, no. 2. pp. 536-542. doi: 10.1016/j.jmaa. 2008.10.042.

2. Мейланов Р. П., Бейбалаев В. Д., Шахбанова М. Р. Прикладные аспекты дробного исчисления. Saarbrücken: Palmarium Academic Publishing, 2012. 135 c.

3. Алхасов А. Б., Мейланов Р. П., Шабанова М. Р. Уравнение теплопроводности в производных дробного порядка // ИФЖ, 2011. Т. 84, № 2. С. 309-317.

4. Самко С. Г., Килбас А. А., Маричев О. И. Интегралы и производные дробного порядка и некоторые их приложения. Минск: Наука и техника, 1987. 688 с.

5. Нахушев А. М. Дробное исчисление и его применение. М.: Физматлит, 2003. 272 с.

6. Tadjeran C., Meerschaert M. M., Scheeffler H.-P. A second-order accurate numerical approximation for the fractional diffusion equation // Journal of Computational Physics, 2006. vol. 213, no. 1. pp. 205-213. doi: 10.1016/j.jcp.2005.08.008.

7. Meerschaert M. M., Tadjeran C. Finite difference approximations for two-sided spacefractional partial differential equations // Applied Numerical Mathematics, 2006. vol.56, no. 1. pp. 80-90. doi: 10.1016/j . apnum.2005.02.008.

8. Лафишева М. М., Шхануков-Лафишев М. Х. Локально-одномерная разностная схема для уравнения диффузии дробного порядка // Ж. вычисл. матем. и матем. физ., 2008. Т. 48 , № 10. С. $1878-1887$.

9. Алиханов А. А. Разностные методы решения краевых задач для волнового уравнения с дробной производной по времени // Вестн. Сам. гос. техн. ун-та. Сер. Физ.-мат. науки, 2008. № 2(17). С. 13-20. doi : 10.14498/vsgtu606.

10. Бейбалаев В. Д. Численный метод решения задачи переноса с двусторонней производной дробного порядка // Вестн. Сам. гос. техн. ун-та. Сер. Физ.-мат. науки, 2009. № 1(18). С. 267-270. doi : 10.14498/vsgtu643.

11. Бейбалаев В. Д. Математическая модель теплопереноса в средах с фрактальной структурой // Матем. моделирование, 2009. Т. 21, № 5. С. 55-62.

12. Таукенова Ф. И., Шхануков-Лафишев М.Х. Разностные методы решения краевых задач для дифференциальных уравнений дробного порядка // Ж. вычисл. матем. и матем. физ., 2006. Т. 46, №10. С. 1871-1881. 
13. Головизнин В. М., Короткин И. А. Методы численного решения некоторых одномерных уравнений с дробными производными // Дифферени. уравнения, 2006. Т. 42, № 7. С. 907913.

14. Бейбалаев В. Д., Абдуллаев И. А., Наврузова К. А., Гаджиева Т. Ю. О разностных методах решения задачи Коши для ОДУ с оператором дробного дифференцирования // Вестник дагестанского государственного университета. Сер. 1. Естественные наукu, 2014. № 6. C. 53-61.

15. Кузнецов Г. В., Шеремет М. А. Разностные методы решения задач теплопроводности. Томск: Томск. политехн. ун-т, 2007. 172 с. 
MSC: 80A22, 26A33

\title{
Modeling of freezing processes by an one-dimensional thermal conductivity equation with fractional differentiation operators
}

\author{
V. D. Beybalaev ${ }^{1,2}$, A. A. Aliverdiev ${ }^{1,2}$, \\ R. A. Magomedov ${ }^{2}$, R. R. Meilanov ${ }^{2}$, E. N. Akhmedov ${ }^{2}$ \\ 1 Dagestan State University, \\ 43a, M. Gadzhiev st., Makhachkala, 367025, Russian Federation. \\ 2 Institute of Geothermal Problems, \\ Dagestan Scientific Center of RAS, \\ 39a, Shamilya av., Makhachkala, 367030, Russian Federation.
}

\begin{abstract}
We have studied the Stefan problem with Caputo fractional order time derivatives. The difference scheme is built. The algorithm and the program for a numerical solution of the Stefan problem with fractional differentiation operator are created. For the given entry conditions and freezing ground parameters we have obtained the space-time temperature dependences for different values of parameter $\alpha$. The functional dependences of the interface motion for the generalized Stefan conditions depending on the value of $\alpha$ are estimated. Finally we have found that the freezing process is slowed down during the transition to fractional derivatives.
\end{abstract}

Keywords: Caputo fractional derivative, fractal structure, Stefan problem, the memory effect, difference scheme, heat conductivity, phase transition, phase boundary.

Received: 28 ${ }^{\text {th }}$ April, $2016 /$ Revised: 10 ${ }^{\text {th }}$ April, $2017 /$ Accepted: $12^{\text {th }}$ June, $2017 /$ First online: $4^{\text {th }}$ July, 2017

Competing interests. We have no competing interests.

\section{Article}

๑ ()(i) The content is published under the terms of the Creative Commons Attribution 4.0 International License (http://creativecommons.org/licenses/by/4.0/)

Please cite this article in press as:

Beybalaev V. D., Aliverdiev A. A., Meilanov R. R., Akhmedov E. N. Modeling of freezing processes by an one-dimensional thermal conductivity equation with fractional differentiation operators, Vestn. Samar. Gos. Tekhn. Univ., Ser. Fiz.-Mat. Nauki [J. Samara State Tech. Univ., Ser. Phys. Math. Sci.], 2017, vol. 21, no. 2, pp. 376-387. doi: 10.14498/vsgtu1492 (In Russian).

\section{Authors' Details:}

Vetlugin D. Beybalaev (D) http://orcid.org/0000-0002-4881-9264

Cand. Phis.\& Math. Sci., Associate Professor; Associate Professor, Dept. of Applied Mathematics ${ }^{1}$; Senior Researcher, Lab. of Mathematical Modeling of Geothermal Objects ${ }^{2}$; e-mail: kaspij_03@mail.ru

Abutrab A. Aliverdiev

Dr. Phis.\& Math. Sci., Professor; Professor, Dept. of Applied Mathematics ${ }^{1}$; Head of the Laboratory, Lab. of Mathematical Modeling of Geothermal Objects ${ }^{2}$; e-mail: aliverdi@mail.ru 
Authors' contributions and responsibilities. Each author has participated in the article concept development and in the manuscript writing. The authors are absolutely responsible for submitting the final manuscript in print. Each author has approved the final version of manuscript.

Funding. This work was partially supported by the Russian Foundation for Basic Research (projects no. 16-08-00067_a).

\section{References}

1. Liu Junyi, Xu Mingyu Some exact solutions to Stefan problems with fractional differential equations, J. Math. Anal. Appl., 2009, vol.351, no.2, pp. 536-542. doi:10.1016/j.jmaa. 2008.10.042.

2. Meilanov R. P., Beybalaev V. D., Shakhbanova M. R. Prikladnye aspekty drobnogo ischisleniia [Applied aspects of fractional calculus]. Saarbrücken, Palmarium Academic Publishing, 2012, 135 pp. (In Russian)

3. Alkhasov A. B., Meilanov R. P., Shabanova M. R. Heat conduction equation in fractionalorder derivatives, Journal of Engineering Physics and Thermophysics, 2011, vol. 84, no. 2, pp. 332-341. doi : 10.1007/s10891-011-0477-9.

4. Samko S. G., Kilbas A. A., Marichev O. I. Integraly i proizvodnye drobnogo poriadka $i$ nekotorye ikh prilozheniia [Integrals and derivatives of fractional order and some of their applications]. Minsk, Nauka i tekhnika, 1987, 688 pp. (In Russian)

5. Nakhushev A. M. Drobnoe ischislenie $i$ ego primenenie [Fractional calculus and its applications]. Moscow, Fizmatlit, 2003, 272 pp. (In Russian)

6. Tadjeran C., Meerschaert M. M., Scheeffler H.-P. A second-order accurate numerical approximation for the fractional diffusion equation, Journal of Computational Physics, 2006, vol. 213, no. 1, pp. 205-213. doi:10.1016/j.jcp.2005.08.008.

7. Meerschaert M. M., Tadjeran C. Finite difference approximations for two-sided spacefractional partial differential equations, Applied Numerical Mathematics, 2006, vol.56, no. 1, pp. 80-90. doi: 10.1016/j. apnum.2005.02.008.

8. Lafisheva M. M., Shkhanukov-Lafishev M. Kh. Locally one-dimensional difference schemes for the fractional order diffusion equation, Comput. Math. Math. Phys., 2008, vol. 48, no. 10, pp. 1875-1884. doi : 10.1134/S0965542508100102.

9. Alikhanov A. A. Difference methods of boundary value problems solution for wave equation equipped with fractional time derivative, Vestn. Samar. Gos. Tekhn. Univ., Ser. Fiz.-Mat. Nauki [J. Samara State Tech. Univ., Ser. Phys. Math. Sci.], 2008, no. 2(17), pp. 13-20 (In Russian). doi: 10.14498/vsgtu606.

10. Beybalaev V. D. Numerical method of solution of the problem on transposition of twosided derivative of the fractional order, Vestn. Samar. Gos. Tekhn. Univ., Ser. Fiz.-Mat. Nauki [J. Samara State Tech. Univ., Ser. Phys. Math. Sci.], 2009, no. 1(18), pp. 267-270 (In Russian). doi : 10.14498/vsgtu643.

11. Beybalayev V. D. Mathematical model of heat transfer in fractal structure mediums, Math. Models Comput. Simul., 2010, vol.2, no.1, pp. 91-97. doi: https://doi.org/10.1134/ S2070048210010096.

Rashid R. Meilanov (D) http://orcid.org/0000-0001-9791-2142

Junior Researcher; Lab. of Mathematical Modeling of Geothermal Objects

Ramazan A. Magomedov (D) http://orcid.org/0000-0001-6224-6215

Senior Researcher; Lab. of Mathematical Modeling of Geothermal Objects; e-mail: ramazan_magomedov@rambler.ru

Enver N. Akhmedov (D) http://orcid.org/0000-0003-4829-6034

Junior Researcher; Lab. of Mathematical Modeling of Geothermal Objects;

e-mail: aen-code@yandex.ru 
12. Taukenova F. I., Shkhanukov-Lafishev M. Kh. Difference methods for solving boundary value problems for fractional differential equations, Comput. Math. Math. Phys., 2006, vol.46, no. 10, pp. 1785-1795. doi: 10.1134/S0965542506100149.

13. Goloviznin V. M., Korotkin I. A. Numerical methods for some one-dimensional equations with fractional derivatives, Differ. Equ., 2006, vol.42, no.7, pp. 967-973. doi: 10.1134/ S0012266106070056.

14. Beybalaev V. D., Abdulaev I. A., Navruzova K. A., Gadgieva T. Yu. Difference methods of solving the Cauchy problem for usual differential equation with operator of fractional differentiation, Vestnik Dagestanskogo gosudarstvennogo universiteta. Ser. 1. Estestvennye nauki, 2014, no. 6, pp. 53-61 (In Russian).

15. Kuznetsov G. V., Sheremet M. A. Raznostnye metody resheniia zadach teploprovodnosti [Difference methods for solving heat conduction problems]. Tomsk, Tomsk Politechn. Univ., 2007, 172 pp. (In Russian) 